Ann. Génét. Sél. anim., r975, 7 (I), 23-33.

\title{
GÉNÉTIQUE DE POPULATION DES CHATS DOMESTIQUES DE MARSEILLE (BOUCHES-DU-RHÔNE, FRANCE)
}

\author{
Ph. DREUX \\ Laboratoire de Zoologie, \\ École normale supérieure, \\ 46, rue d'Ulm, \\ 75230 Paris Cedex 05
}

RÉSUMÉ

\begin{abstract}
Une étude génétique des chats de Marseille a été faite principalement en parcourant les différents quartiers de la ville et en notant le phénotype de chaque animal rencontré. L'application du test de Hardy-Weinberg au gène orange a montré que la population était panmictique. Les fréquences des gènes ont été estimées et trouvées similaires à celles des populations françaises déjà étudiées. Le degré de panachure des chats a été noté. Un certain nombre de petites populations de chats à demi-sauvages ont été observées et trouvées être relativement isolées les unes des autres; les grandes différences de fréquences des gènes entre elles ont été attribuées à une forte influence de l'effet du fondateur.
\end{abstract}

\section{INTRODUCTION}

Depuis la première estimation de la fréquence des gènes dans une population de chats domestiques, faite à Londres par SEARLE (I949) sur une suggestion de J. B. S. HALDANE, les travaux de ce genre se sont multipliés après une période de latence de dix ans. On s'est en effet aperçu que le Chat domestique constituait un intéressant matériel pour l'étude de la sélection, tant naturelle qu'artificielle, à l'échelle du globe terrestre tout entier. C'est en effet une espèce répandue en vastes populations dans le monde, aussi bien dans les villes que dans les campagnes; de plus, il s'agit d'une espèce qui n'est que superficiellement domestiquée et dont la reproduction échappe à l'Homme sauf rares exceptions. C'est donc un matériel très favorable en génétique des populations. 
Jusqu'ici, les sondages ont surtout porté sur les populations de chats des grandes villes, avec peu d'exceptions : chats des fermes d'un district rural de Mayenne (France) (Dreux, I968), ou de petites villes, dans le sud de l'Angleterre (RoBINson et SrL,SON, I969). Les principales investigations ont été faites aux États-Unis et en Europe étendue au bassin de la Méditerranée ; celles qui ont été faites dans le reste du monde sont beaucoup plus dispersées. Les résultats acquis en Europe au cours des années commencent à devenir abondants et cohérents ; on ne citera que ceux qui ont fait l'objet de publications dans les périodiques à large diffusion; en plus de ceux déjà mentionnés, ont déjà été étudiés : Paris (DrEUX, I967 a), Venise (SEARLE, publié dans Dreux, I967 b), York (Metcalfe et Turner, r97I), Chamonix (DreuX, I97I), Vienne (BAXA, I973), La Haye (DREux et LEGEL, I973). Le présent article expose les résultats obtenus dans une autre grande ville : Marseille.

\section{MATÉRIEL E'T MÉTHODES}

Un échantillonnage des phénotypes dans une population de chats, qui permet de calculer les fréquences des gènes, peut être fait en utilisant différents procédés qui ont chacun leurs avantages et leurs inconvénients. Le plus commode est de s'adresser à une clinique vétérinaire où sexe et pelage de tous les animaux apportés par les clients sont soigneusement notés; un exemple récent (DrEUX et LEGEL, I973) a montré que les résultats n'étaient pas toujours faciles à interpréter, parce que ces chats sont sélectionnés par leurs propriétaires sur des critères que l'on connaît mal ; de plus, beaucoup sont stérilisés et ne jouent donc pas de rôle dans la reproduction de la population dans son ensemble.

Une autre méthode est possible quand existe dans la ville une institution du type de la Société Protectrice des Animaux recueillant les chats abandonnés ou errants (DREUx, I967 a) ; il suffit de venir les examiner à intervalles réguliers en s'assurant qu'on ne compte pas le même plusieurs fois ; l'inconvénient est que les animaux abandonnés et errants peuvent parfaitement avoir subi une sélection opposée ou seulement différente qu'il est difficile d'estimer. Le sondage réalisé à Vienne (BAXA, I973) tourne en grande partie cette difficulté en ne portant que sur les animaux qui ont un comportement agressif vis-à-vis de l'Homme et sont donc présumés avec vraisemblance appartenir à la population errante, semi-sauvage.

Une troisième méthode consiste en une enquête directe de porte en porte auprès des propriétaires de chats, en quadrillant une ville par quartiers et par rues ; elle est longue et pénible et présente les mêmes inconvénients que celle de la clientèle vétérinaire ; elle a pourtant été utilisée dans plusieurs sondages en Grande-Bretagne, en cours de publication.

Dans les endroits où les chats sont nombreux et vivent souvent dehors, on peut employer sans y perdre trop de temps la méthode simple qui consiste à parcourir dans la ville un itinéraire systématique en notant tous les animaux que l'on rencontre, en prenant toute précaution utile pour ne pas noter le même plusieurs fois. On échantillonne ainsi la population libre qui participe effectivement à la reproduction (excepté les individus stérilisés, naturellement) et cette méthode serait excellente si elle n'avait un inconvénient gênant : on ne peut déterminer le sexe d'une certaine proportion d'individus trop farouches, ce qui cause des difficultés pour l'étude du gène orange, lié au sexe.

A Marseille, c'est cette dernière méthode qui a été utilisée au cours de plusieurs séjours entre I968 et I973. Lors de chacun, des quartiers différents étaient prospectés, et on ne retournait pas deux fois au même endroit, chose facile car la ville est très étendue. Une seule exception a été faite, qu'on citera plus loin. De plus, deux visites ont été faites, à cinq ans d'intervalle, au refuge de la Société Protectrice des Animaux, où on a pu examiner en tout 74 individus ; cet échantillonnage a été traité séparément du reste, mais n'a pas montré de différences statistiquement significatives, et lui a donc été réuni.

En plusieurs endroits favorables (jardins publics, chantiers, terrains vagues, marchés) on a pu observer de petites populations libres de chats à peu près sauvages dont on a pu voir certainement presque tout l'effectif, à la faveur de distributions de nourriture. Ces petits rassemblements constituaient de véritables sous-populations à l'intérieur desquelles la reproduction devait être panmictique sans beaucoup d'apport extérieur. Une attention particulière leur a été portée, car leur étude pouvait apporter des renseignements sur la structure de la population tout entière. 
En général, ces rassemblements sont temporaires et prennent fin lorsque leur territoire est devenu défavorable pour diverses raisons : lotissement du terrain vague, achèvement du chantier, etc. Toutefois, l'un d'entre eux s'est révélé persistant, celui du Jardin Zoologique; atteignant un effectif supérieur à 70 individus, il a été recensé à cinq ans d'intervalle ; il est extrêmement probable qu'en 1973 il restait bien peu de chats déjà vus en I968. En effet, le renouvellement des populations est rapide et suppose une forte mortalité des individus (DrEUX, 197r).

\section{RÉSULTATS}

\section{I. - Généralités}

Au cours de ces investigations, 550 chats ont été examinés. Cent trente-deux ont été reconnus comme mâles, I7o comme femelles, et le sexe de 248 n'a pu être déterminé. Etant donné que 4I individus de phénotype écaille-de-tortue ont été classés automatiquement comme femelles, alors que les phénotypes orange ont été classés dans les deux sexes au prix de beaucoup d'efforts, la prédominance des femelles ne doit pas être interprétée comme significative et on peut considérer raisonnablement que la proportion des sexes ne diffère pas de $50 \mathrm{p}$. Ioo. Sur ces $55^{\circ}$ individus, I 26 étaient des chatons ou n'avaient pas atteint l'âge adulte ; beaucoup ont été observés se promenant librement et il est par conséquent difficile d'en tirer des conclusions valables au point de vue de la sélection humaine. Il aurait fallu pour cela disposer d'un sondage beaucoup plus important pour comparer ceux observés dans les rues à ceux abandonnés au refuge de la Société Protectrice des Animaux. Tout cet échantillonnage a donc été traité comme homogène.

Le polymorphisme de ces chats a été étudié par rapport aux facteurs génétiques classiquement connus (ROBINSON, I959; SEARLE, I968 ; Committee on nomenclature, I968) :

- $a$, non agouti, épistatique sur $t$,

$-d$, dilution maltaise,

$-l$, persan : poils longs,

$-O$, orange, lié au sexe, épistatique sur $a$; les femelles hétérozygotes ont le phénotype écaille-de-tortue ;

$-S$, panachure blanche irrégulière, semi-dominant à expressivité variable,

$-t^{\text {b }}$, tigré large, allèle de $t^{+}$, tigré ordinaire rayé,

$-W$, blanc dominant, épistatique sur toutes les autres couleurs.

L'allèle $T$ a au locus $t$ (tigré type Abyssinie) n'a pas été rencontré dans cet échantillonnage. Quatre chats siamois $c^{s}$ ont été vus et ont été exclus de l'ensemble car il s'agissait d'individus très surveillés ou castrés ne prenant pas part à la reproduction ; il est toutefois manifeste que cet allèle se répandra inévitablement par la suite dans la population. On a observé 7 individus " chinchilla " (sur phénotype tigré) et 8 " argentés " (sur phénotype non-agouti); le déterminisme de ce facteur considéré autrefois comme allèle du locus $c$ étant encore à l'étude, il n'a pas été étudié au point de vue de la fréquence génique (RoBInson, comm. pers.). Au point de vue des anomalies squelettiques, seuls deux chats à queue tordue (kinky-tail) ont été observés, mais aucun syndactyle ou polydactyle ; beaucoup d'individus n'ayant pas été examinés de près, cette conclusion n'a rien de très affirmatif. 


\section{2. - Test de la panmixie; gène orange $\mathrm{O}$}

Trois individus blancs dominants $W$, une femelle et deux de sexe non identifié doivent être exclus de ce classement qui comporte donc $132 \widehat{\delta} \sigma^{\circ}$, 169 우우 et 246 non identifiés. Comme la classification au point de vue du gène orange est très importante, tous les individus orange ont été suivis jusqu'à la détermination relativement certaine de leur sexe ; 24 étaient mâles et 2 femelles. D'autre part, 4 I femelles écaillede-tortue ont été repérées ; il n'y a jamais eu de difficultés à reconnaître ce phénotype. Il a parfois été suggéré (en particulier par METCALFE et TURNER, I97I) que le déficit fréquent des phénotypes écaille-de-tortue était dû au fait que de faibles plages orange sur une femelle agouti peuvent passer inaperçues ; une telle erreur nous paraît peu vraisemblable et on invoquerait de préférence un manque de pénétrance du gène orange dans les génotypes $O /+$. L'explication par une sélection différentielle chez les deux sexes, proposée par ces auteurs, paraît plus plausible.

\section{TABLEAU I}

Distributions observées et calculées (entre parenthèses) des phénotypes correspondant au gène orange dans la population de chats de Marseille

\begin{tabular}{c|c|c|c}
\hline \hline Genotypes & $+1-$ ou $+1+$ & $O /+$ & $O /-$ ou $O / O$ \\
\hline Mâles (273) & $249(250,1)$ & 0 & $24(22,9)$ \\
\hline Femelles (274) & $231(229,9)$ & $-\frac{21(42,2)}{2(1,8)}$ \\
\hline
\end{tabular}

En supposant l'égalité de fréquence des deux sexes dans la population, comme il a été dit plus haut, on peut admettre qu'il existe 69 gènes orange sur 820 loci, ce qui donne une fréquence du gène $O$ de $0,084 \mathrm{I}$. On a calculé sur cette base les fréquences théoriques des différents phénotypes conformément à la loi de HardyWeinberg. Elles sont présentées dans le tableau I, en comparaison avec les fréquences observées ; 1'accord s'y révèle excellent (le $\chi^{2}$ est très inférieur à I pour 3 degrés de liberté) si les hypothèses faites sur le sex-ratio sont exactes, et on peut admettre l'hypothèse de la panmixie pour le gène orange dans l'ensemble de la population ; on y a postulé l'existence de 273 ô ô et 274 우오 (tabl. I).

\section{3. - Fréquence des gènes}

La population de Marseille ayant été trouvée panmictique par rapport au gène $O$, il est assez vraisemblable de supposer qu'elle l'est aussi par rapport aux autres gènes examinés, ce qui permet de calculer leurs fréquences par application de la loi de Hardy-Weinberg. Ces fréquences sont données dans le tableau 2 où 1'on a fait aussi figurer celle de $O$. 
TABLEAU 2

Fréquence des gènes dans la population des chats de Marseille

\begin{tabular}{|c|c|c|c|c|}
\hline Allèles (1) & $\begin{array}{c}\text { Nombres } \\
\text { classifiables }\end{array}$ & $\begin{array}{c}\text { Effectifs } \\
\text { phénotypiques (1) }\end{array}$ & $\begin{array}{c}\text { Fréquences } \\
\text { phénotypiques } \\
(\text { en } \%)\end{array}$ & $\begin{array}{c}\text { Fréquences } \\
\text { géniques }\end{array}$ \\
\hline$a$ & 521 & 273 & 52,4 & 0,72 \\
\hline$d$ & 547 & 65 & 11,9 & 0,34 \\
\hline$l$ & 550 & 41 & 7,4 & 0,27 \\
\hline$O$ & 547 & - & - & 0,08 \\
\hline$t^{+}$ & 266 & 144 & 54,1 & 0,32 \\
\hline$t^{\mathrm{b}}$ & 266 & 122 & 45,9 & 0,68 \\
\hline$W$ & 550 & 3 & 0,5 & 0,003 \\
\hline
\end{tabular}

(1) Les effectifs phénotypiques observés sont ceux de l'homozygote pour les gènes récessifs (exemple : $a a)$, celui de l'homozygote ou de l'hétérozygote pour les gènes dominants (exemple [W]).

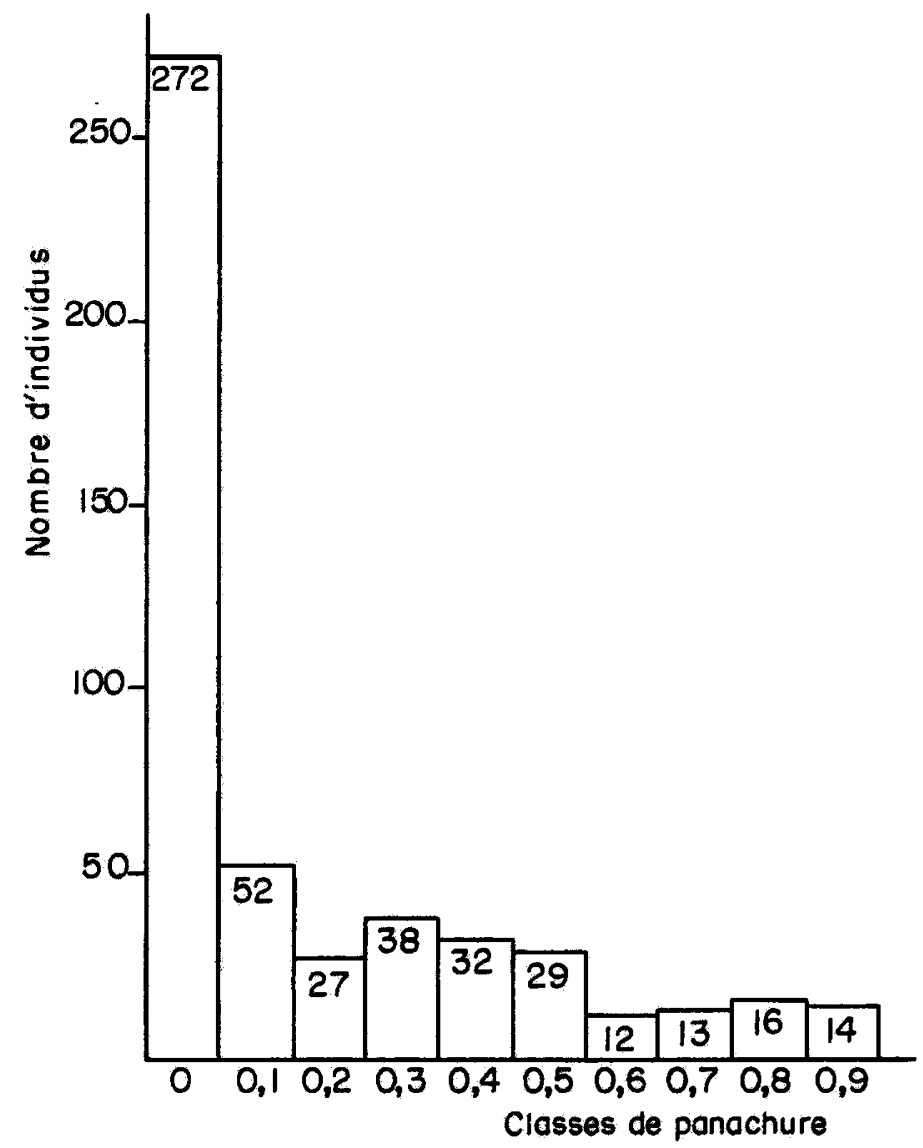

FIG. x. - Rëpartition des degrès de panachure chez les chats de Marseille 


\section{4. - Panachure}

Tous les chats qui ne présentaient pas le phénotype blanc dominant $[W]$ ont été classés au moyen de l'échelle de panachure précédemment définie (DREUx, I967 $a$ ) dans des catégories de 0 à 0,9 , les nombres exprimant approximativement la fraction de surface du corps de couleur blanche. On a aussi noté à tout hasard chez les individus non-agouti (les seuls pour lesquels ce soit bien visible) et dépourvus de la panachure irrégulière, la présence sous la gorge ou sous le ventre (ou les deux) d'une petite touffe de poils blancs, déjà dénommée panachure $\varepsilon$. Elle était sûrement présente chez 42 chats au moins sur un total de ro7. Le déterminisme de cette panachure discrète est encore mal connu, mais certainement héréditaire : en effet, la sélection l'a fait disparaître des races d'exposition. Au contraire, on sait que la panachure irrégulière est due à un gène semi-dominant $S$, les homozygotes $S S$ étant en moyenne plus largement panachés de blanc que les hétérozygotes $S s$.

La proportion d'individus sans panachure irrégulière, donc de génotype ss permet de calculer la fréquence du gène $S$ dans la population. L'allèle $s$ non panaché est homozygote chez 272 individus sur 547 , il a donc une fréquence de 0,705 et celle de l'allèle $S$ est par suite de 0,295 . Si l'on suppose que l'échantillon étudié se conforme à la loi de Hardy-Weinberg, les individus de génotype $S S$ peuvent être estimés en nombre voisin de 48 . Cette valeur est en assez bon accord avec l'histogramme de distribution de la panachure qui montre une certaine discontinuité vers la classe 0,6 ; on pourrait admettre avec assez de vraisemblance qu'une partie des individus de la classe o,6 est $S s$, l'autre $S S$ et que tous les individus des classes supérieures sont de génotype $S S$ (fig. I).

\section{5. - Populations semi-isolées}

Comme il a déjà été exposé plus haut, on a rencontré à Marseille un certain nombre de petits isolats de chats à demi-sauvages, vivant en liberté dans des biotopes favorables, nourris occasionnellement par les gens du voisinage chez lesquels ils n'habitaient pas. Il a paru intéressant d'étudier ces isolats dans la mesure du possible ; on en a vu une dizaine dont l'effectif repéré variait de 8 à plus de 70 . Ces chats étaient en général très difficiles à approcher et leur sexe difficile à déterminer ; on a supposé que dans ces isolats, la proportion de mâles était de 0,5 comme dans le reste de la population. Leur localisation géographique était la suivante (fig. 2) :

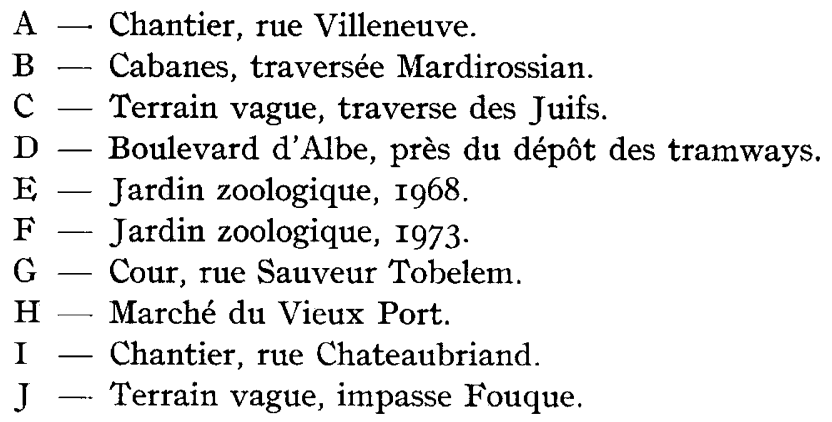




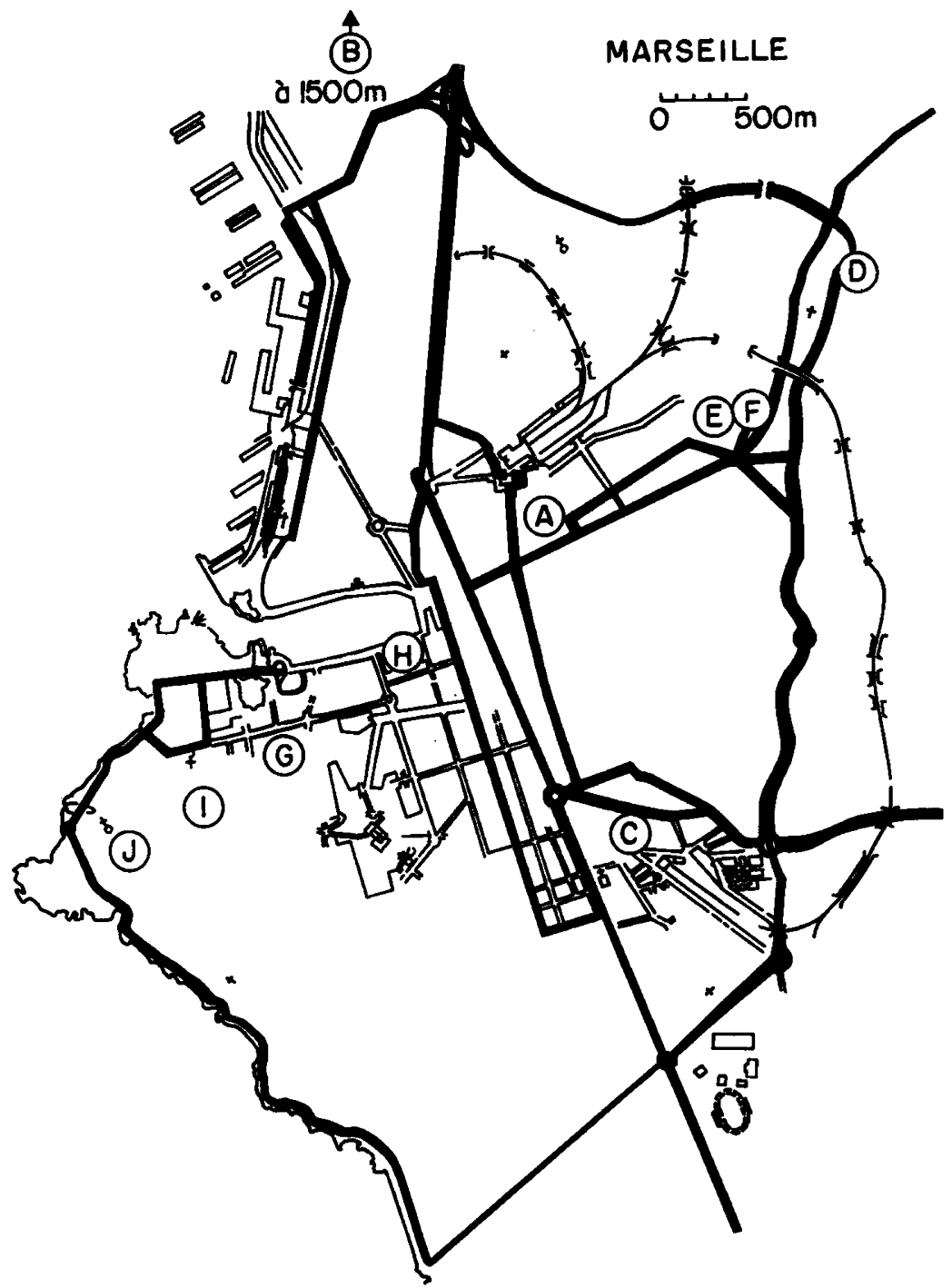

FIG. 2. - Distribution spatiale des rassemblements de chats étudies dans la ville de Marseille

Malgré les effectifs le plus souvent faibles, on a calculé pour chacun de ces isolats les valeurs approximatives des fréquences des gènes $O, a$ et $t^{\mathrm{b}}$ qui sont données dans le tableau 3. Le nombre total de chats qui y ont été observés est de 250 soit près de la moitié de l'échantillonnage de Marseille. Les fréquences géniques sont très variables comme il fallait s'y attendre, montrant certainement une grande influence de l'effet du fondateur au moment de la constitution de ces isolats dont la plupart sont temporaires, liés à l'existence momentanée d'un biotope favorable. L'urbanisation tend à les faire disparaître dans beaucoup de quartiers. 
TABLEAU 3

Fréquences approximatives des gènes $\mathrm{O}$, a et th dans quelques rassemblements de chats semi-sauvages à Marseille

\begin{tabular}{|c|c|c|c|c|}
\hline Lieu & Effectif total & $\begin{array}{c}\text { Fréquence } \\
\text { de } O\end{array}$ & $\begin{array}{l}\text { Fréquence } \\
\text { de } a\end{array}$ & $\begin{array}{c}\text { Fréquence } \\
\text { de } t^{\mathbf{b}}\end{array}$ \\
\hline A & 17 & 0,28 & 0,68 & 0,95 \\
\hline B & 11 & 0 & 0,84 & $?(1)$ \\
\hline C & 15 & 0,09 & 0,84 & 0,71 \\
\hline D & 9 & 0,21 & 0,84 & 0,87 \\
\hline E & 72 & 0,01 & 0,70 & 0,69 \\
\hline $\mathrm{F}$ & 71 & 0,02 & 0,80 & 0,69 \\
\hline G & 11 & 0 & 0,74 & $?\left({ }^{1}\right)$ \\
\hline $\mathrm{H}$ & 8 & 0,38 & 0,84 & 1,00 \\
\hline I & 17 & 0,30 & 0,76 & 0,77 \\
\hline$J$ & 19 & 0,41 & 0,82 & 0,78 \\
\hline
\end{tabular}

(') L'homozygote récessif n'a pas été observé,

\section{DISCUSSION}

La discussion des résultats portera sur deux points principaux.

\section{I. - Comparaison avec les autres populations d'Europe}

Le tableau 4 qui résume les données publiées jusqu'ici sur les populations de chats en Europe montre que celle de Marseille se rapproche extrêmement de celles de Paris et de Chamonix, la population rurale étudiée en Mayenne montrant une fréquence plus forte de $O$ et une fréquence plus faible de $a, t^{\mathrm{b}}$ et $l$; ceci avait été interprété comme résultant d'une différence de sélection en milieu rural par rapport au milieu urbain. L,es populations d'Angleterre sont surtout caractérisées par une fréquence élevée du gène orange. Les populations plus orientales (Venise, Vienne) montrent surtout une baisse importante des allèles $a$ et $t^{\mathbf{b}}$, qui semble confirmée par des résultats en cours de publication (ToDD, 1974).

Il était assez vraisemblable que la situation de Marseille, grand port offrant un trafic important avec les régions orientales de la Méditerranée, pouvait se traduire sur sa population de chats. Il n'en est rien : les trois populations de Marseille, Paris et Chamonix sont extrêmement similaires ; on peut penser que les échanges de chats par bateaux sont très rares ou en tout cas n'exercent pas d'influence sur une population nombreuse et bien établie.

Malgré une certaine homogénéité des populations européennes, on commence 
d'autre part à voir se dessiner une variation géographique pour la fréquence de certains allèles, surtout $t^{\mathrm{b}}$ et $a$ vers l'est et $O$ vers le nord. Des sondages récents en voie de publication semblent bien confirmer ces vues et il est possible qu'il soit alors nécessaire de réviser l'interprétation des différences entre la population rurale de Mayenne et les populations urbaines de France en tenant compte de sa position géographique.

\section{TABLEAU 4}

Fréquence des gènes dans les populations de chats d'Europe occidentale

\begin{tabular}{|c|c|c|c|c|c|c|c|c|c|c|}
\hline & 总 & 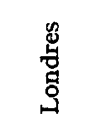 & 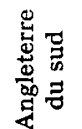 & 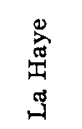 & 莺 & $\begin{array}{l}\text { : } \\
\text { 总 } \\
\text { 严 }\end{array}$ & 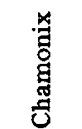 & 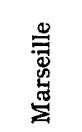 & 兽 & 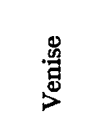 \\
\hline$a$ & 0,81 & 0,76 & 0,80 & 0,65 & 0,71 & 0,64 & 0,75 & 0,72 & 0,57 & 0,58 \\
\hline$d$ & 0,27 & 0,14 & 0,26 & 0,26 & 0,33 & 0,29 & 0,40 & 0,34 & 0,22 & 0,35 \\
\hline$l$ & $?$ & $0,33\left({ }^{1}\right)$ & 0,32 & 0,16 & 0,24 & 0,17 & 0,32 & 0,27 & - & $?$ \\
\hline$O$ & 0,20 & 0,11 & 0,19 & 0,21 & 0,06 & 0,15 & 0,10 & 0,08 & 0,10 & 0,06 \\
\hline$S$ & $0,34\left(^{1}\right)$ & 0,37 (1) $^{\mathbf{1}}$ & 0,31 & 0,36 & 0,24 & 0,39 & 0,22 & 0,29 & 0,16 & $0,27\left(^{1}\right)$ \\
\hline$T^{\mathrm{a}}$ & - & 0,002 & - & 0,002 & 0,001 & - & - & - & 0,001 & - \\
\hline$t^{+}$ & 0,22 & 0,19 & 0,16 & 0,34 & 0,22 & 0,39 & 0,31 & 0,32 & 0,71 & 0,52 \\
\hline$t^{b}$ & 0,78 & 0,81 & 0,84 & 0,66 & 0,78 & 0,61 & 0,69 & 0,68 & 0,29 & 0,48 \\
\hline$W$ & 0,012 & 0,004 & 0,014 & 0,019 & 0,011 & 0,006 & 0,014 & 0,003 & 0,001 & 0,003 \\
\hline
\end{tabular}

(1) Valeurs calculées d'après les données des auteurs.

\section{2. - Structure de la population}

L'examen des petites populations plus ou moins isolées et à demi-sauvages que l'on rencontre à Marseille a donné des résultats intéressants qui malheureusement ne portent pas sur des effectifs suffisants pour une interprétation complète.

Leur forte proportion dans l'échantillonnage total montre cependant qu'au moins dans des villes du genre de Marseille il existe de nombreux chats à demisauvages, vivant en petites populations plus ou moins isolées, et qui doivent former la masse principale du pool génique. Il y a certainement des échanges de l'une à l'autre, surtout par l'intermédiaire d'animaux plus domestiques et plus sédentaires, mais ils doivent être assez limités. La disparition d'un de leưrs biotopes favorables disperse la population qui l'habitait dans la région avoisinante, où les individus peuvent retrouver un nouveau territoire, mais beaucoup ne doivent pas $y$ arriver et disparaissent.

La très grande dispersion des fréquences géniques poul les gènes $0, a$ et $t^{\mathrm{b}}$ dans ces petites populations montre bien le rôle du hasard dans leur fondation. On a cherché, en reportant ces localités sur une carte de la ville, si une influence géographique s'exerçait sur ces fréquences, en d'autres termes si on pouvait y déceler des clines (fig. 2). Il ne semble pas que ce soit le cas, quoique la plupart des fréquences 
élevées de $O$ se rencontrent dans le sud de la ville; mais les données sont vraiment trop peu nombreuses pour pouvoir amener à des conclusions certaines. Il faudrait toutefois essayer d'entreprendre sur une ville aussi favorable une vaste enquête beaucoup plus serrée et beaucoup plus suivie ; elle pourrait donner des renseignements intéressants sur la structure de la population globale d'une grande cité.

On doit faire une mention particulière du rassemblement de chats qui existe au jardin zoologique, le plus important en effectif. Examiné à cinq ans d'intervalle, cet effectif semble être étonnamment stable : 72 chats vus en I968, 7 I en I973, pour un effort d'investigation tout à fait comparable. Cette population est également restée identique au point de vue des fréquences des gènes $O$ et $t^{\mathrm{b}}$, avec une forte augmentation de la fréquence de $a$. Il serait intéressant de la suivre pendant longtemps et de voir si cette stabilité se maintient.

On a enfin essayé de voir si les fréquences géniques étaient les mêmes dans l'ensemble de ces petits rassemblements et dans le reste de la population. Chez ces chats en semi-liberté, on trouve les fréquences suivantes : pour 0,0, II ; pour $a: 0,77$; pour $t^{\mathrm{b}}: 0,73$. Chez les chats rencontrés isolément, elles étaient respectivement de 0,$06 ; 0,69$ et 0,63 . Les différences sont appréciables, mais non statistiquement significatives; elles pourraient cependant constituer un argument en faveur de la meilleure survie en environnement urbain des individus $a a$ et $t^{b} t^{b}$, mais cette interprétation est assez hypothétique.

\section{CONCLUSION}

Contrairement à ce qui avait été envisagé en entreprenant cette étude, la situation de Marseille, grand port maritime présentant beaucoup d'échanges avec des régions orientales, ne semble avoir absolument pas influencé la population de chats qui s'y trouve. Celle-ci appartient au type moyen de l'Europe occidentale et elle est particulièrement proche par ses fréquences géniques de celles de Paris et de Chamonix.

L'examen attentif de la population montre que beaucoup de biotopes favorables sont occupés par des colonies plus ou moins nombreuses de chats en liberté. Ces colonies n'ont que peu d'échanges les unes avec les autres et le principe du fondateur y joue beaucoup, ce qui fait qu'elles diffèrent très fortement les unes des autres par leurs fréquences géniques. La population de Marseille - et probablement celles de beaucoup de villes analogues - se présente ainsi comme un ensemble de petites colonies très différentes, assez éphémères d'ailleurs car liées à l'existence temporaire d'un biotope favorable, et reliées par des migrations de gènes à travers des individus peu nombreux.

Une étude entreprise sur une grande échelle pourrait donner des résultats intéressants sur la structure réelle d'une telle population et permettre en particulier d'apprécier le territoire efficace des individus au point de vue de la reproduction. La structure très hétérogène déjà mise en évidence souligne le danger qu'il y a à fonder l'étude de la population de chats d'une ville sur un échantillonnage de trop faible effectif. 


\section{SUMMARY}

\section{POPULATION GENETICS OF AT MARSEILLE'S DOMESTIC CATS}

A genetic study of Marseille's cats was made essentially by walking in different districts of the town and checking each animal encountered. The application of Hardy-Weinberg's test to the orange gene showed that the population was randomly mated. Gene frequencies were estimated and found similar to those of the french populations already studied. The amount of white-spotting in the cats were checked. Many small populations of semiferal cats were observed and found to be somewhat isolated from each other; the great differences of gene frequencies were attributed to a strong founder effect.

\section{RÉFÉRENCES BIBLIOGRAPHIQUES}

BAXA H., 1973. Gene frequencies in stray cat populations from Vienna. Genetica, 44, 25-30.

COMMITTEE ON STANDARDized GENETIC NOMENCLATURE FOR CATS, 1968. Standardized genetic nomenclature for the domestic cat. J. Hered., 59, 39-40.

DreuX Ph., I967 a. Gene frequencies in the cat population of Paris. J. Hered., 58, 89-92.

Dreux Ph., r 967 b. Génétique des populations de chats domestiques en Europe occidentale. Ann. Génét., 10, I4I-I45.

DreUX Ph., 1968. Gene frequencies in the cat population of a french rural district. J. Hered., 59, 37-39.

Dreux Ph., I97I. Génétique des populations de chats domestiques dans la vallée de Chamonix (HauteSavoie). Ann. Génét. Sél. anim., 8, r45-15I.

Dreux Ph., Legel J. C., 1973. Gene frequencies in the cat population of The Hague. J. Hered., 64, 337-339.

Metcalfe J. A., Turner J. R. G., I97I. Gene frequencies in the domestic cats of York : evidence of selection. Heredity, 26, 259-268.

Robinson R., I959. Genetics of the domestic cat. Biblio. Genet., 8, 273-362.

Robinson R., Silson M., 1969. Mutant gene frequencies in cats of southern England. Theor. Appl. Genet., 39, 326-329.

SeARLE A. G., 1949. Gene frequencies in London's cats. J. Genet., 49, 214-220.

SEARLE A. G., I968. Comparative genetics of coat colour in Mammals. Logos Press/Academic Press, 308 p., New-York and London.

Topd N. B., 1974. Communication personnelle. 